\title{
A solitary benign lymphoid polyp of the rectum in a 51 year old woman
}

\author{
J Lloyd, A Darzi, J Teare, R D Goldin
}

\begin{abstract}
A 51 year old woman presented with rectal bleeding from a mass lying just above the dentate line. Multiple biopsies were taken before the diagnosis of a benign lymphoid polyp was made. This is a rare condition that more commonly presents in children and poses a diagnostic challenge. (f Clin Pathol 1997;50:1034-1035)
\end{abstract}

Keywords: large bowel; lymphoid hyperplasia; polyp

Intestinal lymphoid hyperplasia is a localised or widespread benign proliferation of lymphoid tissue within the intestinal wall, producing single or multiple lesions. Clinically it takes the form of benign lymphoid polyps. It has been described at all levels of the gastrointestinal tract with the majority of cases involving the small bowel and rectum. While relatively common in children, benign lymphoid hyperplasia of the colon and rectum is rarely described in adults. Important considerations in this condition are the distinction from malignant lymphoma and the possible association with subsequent development of malignant lymphoma.

We report a case of solitary benign lymphoid polyp of the colon in a 51 year old woman, which posed a diagnostic problem.

\section{Case report}

The patient, a 51 year old Filipino woman, was referred to St Mary's Hospital in October 1995 with recent onset fresh bleeding per rectum mixed with faeces, associated with tenesmus and perineal pain. Rectal examination revealed a polypoid mass arising just above the dentate line. Initial biopsies of the lesion showed large bowel mucosa with an infiltrate composed predominantly of lymphocytes. One section contained scattered atypical lymphocytes and the possibility that this was a rectal lymphoma was raised. Subsequent biopsies showed rectal mucosa in which the submucosa was expanded by large lymphoid follicles with reactive germinal centres and cuffs of mantle cells. The follicles were subdivided by sinuses. Immunohistochemical staining revealed the infiltrate to be a mixed population of $B$ and $T$ lymphocytes, and macrophages. A diagnosis of a benign lymphoid polyp was made. Transanal resection of the polypoid mass was performed early in 1996.

The definitive specimen was a fleshy mass $(3 \times 3 \times 2.5 \mathrm{~cm})$ partially covered by mucosa, which had a homogeneous pale cut surface. Histological examination showed infiltration of all layers of the wall of the large bowel, with extension through the muscularis externa by

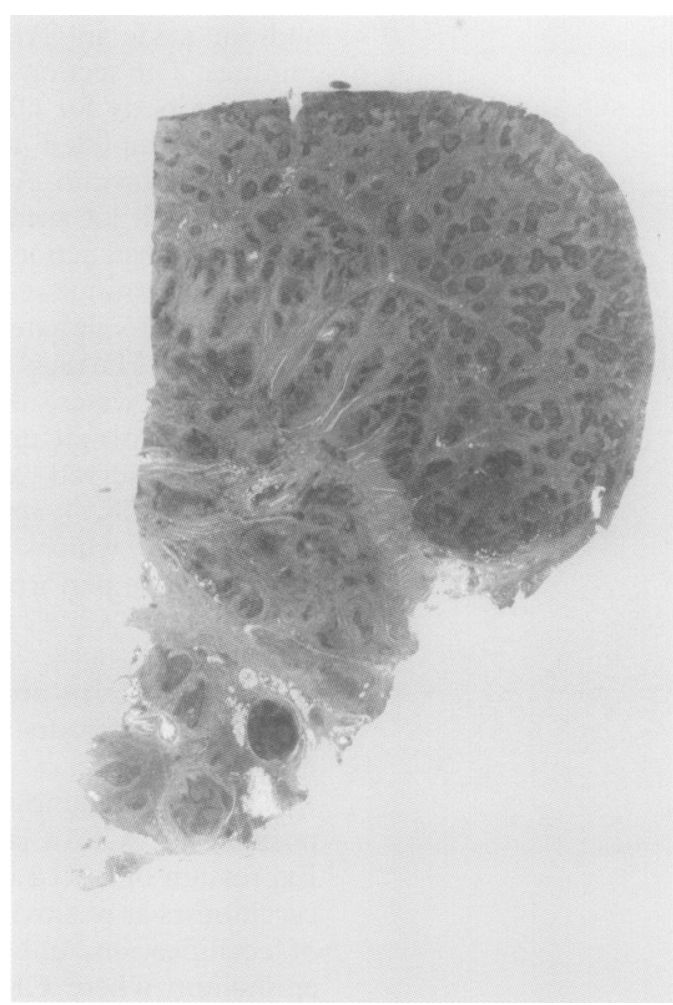

Figure 1 Low power view of lymphoid polyp showing infiltration of lymphoid follicles through all layers of the bowel wall (haematoxylin and eosin; original magnification $\times 10$ ).

numerous lymphoid follicles of varying sizes, with eccentric mantle zones and prominent germinal centres (figs 1 and 2). The intervening connective tissue contained a plasma cell rich infiltrate. The follicles had a high proliferative rate (as assessed by $\mathrm{Ki}-67$ staining) and low level bcl-2 expression. Malignant lymphoma was excluded with polymerase chain reaction light chain restriction analysis.

\section{Discussion}

Lymphocytes are normally present within colonic mucosa, both diffusely distributed and organised into lymphoid follicles. The lymphoid follicles may be confined wholly within the lamina propria or within the submucosa, or they may extend across the muscularis mucosa and occupy both (forming a lymphoglandular complex). The surface epithelium overlying a lymphoid follicle usually consists of cuboidal or low columnar epithelium that may be heavily infiltrated by small lymphocytes. " $M$ " cells serve as conduits for the transport of luminal antigens to the underlying lymphoid tissue. ${ }^{1}$

Benign lymphoid hyperplasia of the intestine has been described for all parts of the gastrointestinal tract and is defined as a benign 


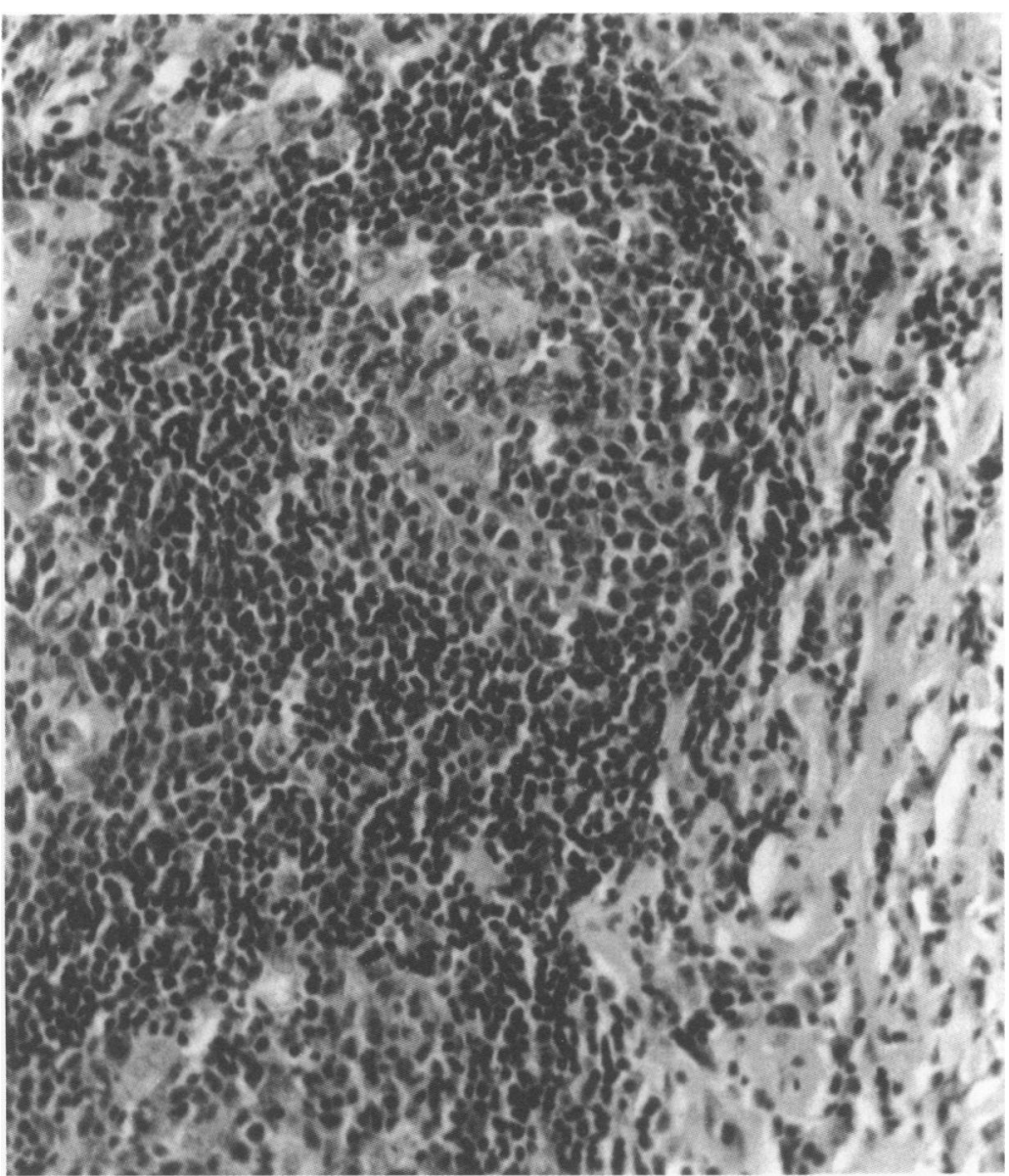

Figure 2 Representative follicle at high power showing the reactive germinal centre (haematoxylin and eosin; original magnification $\times 20$ ).

proliferation of the lymphoid tissue in the intestinal wall forming a space occupying lesion. The proliferation may be localised or extensive, producing single or multiple lesions. ${ }^{2}$ Reactive hyperplasia resulting from specific infections, such as infectious mononucleosis, or inflammatory disorders, such as Crohn's disease, diversion colitis or sarcoidosis are excluded.

The condition is often an incidental finding, hence its true prevalence is unknown. Most cases described involve the jejunum, ileum, and rectum. While relatively frequent in childhood, benign lymphoid hyperplasia of the colon is rare in adults. ${ }^{3}$ Almost all are benign. A benign lymphoid polyp must be distinguished clinically from an adenomatous polyp and from malignant lymphoma. Our case, in which the lesion had ulcerated, was diagnosed clinically as a rectal carcinoma.

Lesions in the small bowel may present with intussusception or occult anaemia. Colorectal lesions more commonly present with bleeding, and rectal lesions may present with prolapse. Other presentations include weight loss, diarrhoea, and abdominal pain. The association of benign lymphoid hyperplasia with primary immunodeficiency syndromes is recognised and the possibility of hypogammaglobulinaemia should be considered. Symptomatic lesions can be surgically excised, effecting a cure.

Macroscopically the lesions are variable in appearance, ranging from tiny sessile polyps to large, pedunculated masses or ulcerated nodules. Microscopically well formed follicles are seen with prominent germinal centres. The lymphocytes show orderly maturation and plasma cells are often seen at the periphery of the clearly separated follicles. All these features were seen in this case.

This appearance may involve only the mucosa and submucosa or may be present throughout the entire thickness of the bowel wall when it may become difficult to distinguish between benign hyperplasia and malignant lymphoma. The polymorphous nature of the infiltrate, as confirmed by immunohistochemical methods, and the presence of true germinal centres, helps to confirm that the lesion is benign. Monoclonal infiltrates are thought, in most cases, to have low malignant potential but should raise suspicion of malignancy. ${ }^{4}$ Lymphoepithelial lesions in which glands and crypts are invaded by lymphoid cells is a feature of malignant follicular centre cell lymphoma. Cytogenetic analysis, demonstrating the proportion of aneuploid cells, may play a role in difficult cases.

The aetiology of benign lymphoid hyperplasia is unknown. It may be a reactive response to antigenic stimulation, possibly a viral infection. Solitary lesions may form in response to prolonged local stimulation of the mucosal immune system. In immunodeficiency syndromes there may be stimulation from invading microorganisms or loss of control of normal lymphocyte proliferation.

The distinction of benign lymphoid polyps from malignant lymphomas can be difficult. The gastrointestinal tract is the most common site of presentation for extranodal lymphoma, and malignant lymphomas of the gastrointestinal tract may manifest as multiple lymphoid polyposis. However, follicular lymphomas of the gastrointestinal tract are rare. ${ }^{5}$ While most benign proliferations do not extend into the muscularis externa it is apparent (as our case demonstrates) that the depth of infiltration is not a reliable feature for distinguishing benign from malignant lymphoid proliferations. Immunohistochemistry and polymerase chain reaction for light chain restriction may be essential to confirm the benign nature of these lesions.

We thank Professor P G Issaacson for his expert opinion on this case.

1 Sternberg SS. Histology for pathologists. New York: Raven Press, 1996:577-9.

2 Fenoglio-Preiser CM, Pascal RR, Perzin KH. Tumours of the intestines, Washington DC: Armed Forces Institute of Pathology 1990:391-6.

3 Benchimol D, Frileux P, Herve de Sigalony JP, Parc R. Benign lymphoid polyposis of the colon. Report of a case in an adult. Int $\mathcal{F}$ Colorectal Dis 1991;6:165-8.

4 Burke JS, Sheibani K, Nathwani BN, Winberg CD, Rappaport H. Monoclonal small (well-differentiated) lymphocytic proliferations of the gastro-intestinal tract resembling malignant potential. Hum Pathol 1987;18:1238-45.

5 LeBrun DP, Kamel OW, Cleary ML, Dorfman RF, Warnke RA. Follicular lymphomas of the gastro-intestinal tract. Pathologic features in 31 cases and bcl-2 oncogenic protein expression. Am f Pathol 1992;140:1327-35. 